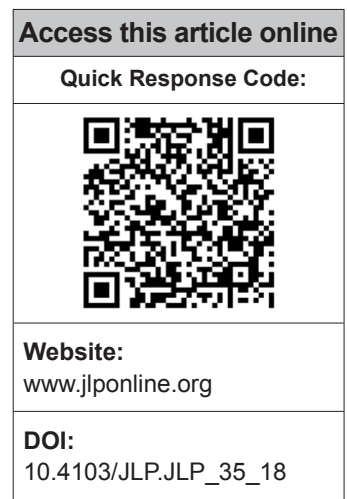

Departments of Pathology, University College of Medical Sciences and

Guru Teg Bahadur Hospital, ${ }^{1}$ Department of Pathology, Max Hospital Saket, New Delhi, India

Address for correspondence: Dr. Mrinalini Kotru,

Department of

Pathology, University College of Medical

Sciences and Guru Teg Bahadur Hospital, New Delhi - 110 095, India.

E-mail: mrinalini.kotru@ gmail.com

Submission: 04-03-2018 Accepted: 05-08-2018

\title{
Correlation of expression of aberrant immunophenotypic markers in T-ALL with its morphology: A pilot study
}

\author{
Neha Garg, Mrinalini Kotru, Dilip Kumar ${ }^{1}$, Rajesh Pathak, Meera Sikka
}

\section{Abstract:}

INTRODUCTION: Aberrant expression of immunophenotypic markers is commonly found in patients of acute leukemia. T-ALL also shows aberrant markers such as CD13, CD33, CD117, CD10, and CD79a. Morphologically, T-ALL has been categorized into L1, L2, and L3 subtypes. Till now, no study has been done to correlate these markers with morphological features of T-ALL. This study aimed to correlate the expression of aberrant immunophenotypic markers with morphology in T-ALL.

MATERIALS AND METHODS: All the cases of T-ALL diagnosed by flow cytometry over a period of $2^{1 / 2}$ year were taken out from the records of Hematology Section of Department of Pathology of University College of Medical Science and Guru Teg Bahadur Hospital and Max Hospital, Saket. Their peripheral blood smear was screened to correlate the morphology of blasts with the expression of aberrant markers.

RESULTS: A total of 40 cases of T-ALL were identified during $2 \frac{1}{2}$ year period of our study. Morphological correlation was available for 23 cases. Aberrant expression of CD10 was present in $6(35.3 \%)$ cases, CD79a in $9(47.36 \%)$ cases, CD117 in $5(42.28 \%)$ cases and myeloid antigen CD33 in $5(38.46 \%)$ cases. CD117 and CD33-positive cases showed L2 morphology with the presence of convolutions, while cases with expression of CD79a had L1 morphology with absent-slight convolutions. CD10-positive cases had L1/L2 morphology with absent occasionally present convolutions.

CONCLUSIONS: There seems to be an association of aberrant markers with L1 and L2 morphology. However, this needs to be tested for statistical significance on a larger sample size.

Key words:

Acute lymphoblastic leukemias, lymphoid cell neoplasms, neoplasia, T cell neoplasms

\section{Introduction}

A berrant expression of immumophenotypic markers is commonly found in patients of acute leukemia. T-cell acute lymphoblastic leukemia (T-ALL) also shows aberrant markers such as CD13, CD33, CD117, CD10, and CD79a. Morphologically, ALL has been categorized into L1, L2, and L3 subtypes. ${ }^{[1]}$ Till now, no study has been done to correlate these markers with morphological features of T-ALL. In an

This is an open access journal, and articles are distributed under the terms of the Creative Commons Attribution-NonCommercial-ShareAlike 4.0 License, which allows others to remix, tweak, and build upon the work non-commercially, as long as appropriate credit is given and the new creations are licensed under the identical terms.

For reprints contact: reprints@medknow.com era of flow cytometry, morphology is assuming a backseat; nevertheless, it is interesting to identify such associations. This study aimed to correlate the expression of aberrant immunophenotypic markers with morphology in T-ALL.

\section{Materials and Methods}

This descriptive study was done in collaboration with the Hematology Section of two tertiary care centers in Delhi. All the cases of T-ALL diagnosed by flow cytometry over a period of $2^{1 / 2}$ years were taken out from the records. Their peripheral blood smear was

How to cite this article: Garg N, Kotru M, Kumar D, Pathak R, Sikka M. Correlation of expression of aberrant immunophenotypic markers in T-ALL with its morphology: A pilot study. J Lab Physicians 2018;10:410-3. 
screened to correlate the morphology of blasts with the expression of aberrant markers. The diagnosis of T-ALL was made by expression of $\mathrm{CCD} 3$ in $>10 \%$ of blasts. A total of 40 cases of T-ALL were identified during 21/2-year-period of our study. In all the cases, peripheral smear stained by Wright's stain was examined for morphology of the blasts. They were categorized into L1, L2, and L3 on the basis of French-American-British (FAB) classification. ${ }^{[1]}$ L1 blasts are smaller in size, have coarse chromatin and homogenous nuclei. L2 blasts are larger than L1 blasts and are characterized by nuclear heterogeneity. L3 blasts are also larger than L1 blasts, have homogeneous nuclei and are characterized by the prominence of vacuoles inside of the cell. ${ }^{[1]}$ Further, immunophenotyping was done on flow cytometer (Beckman coulter, FC500). The antibody expression of CD10, CD79a, CD33, and CD117 was studied. Expression of aberrant markers and their correlation with morphology in T-ALL was then analyzed statistically.

\section{Results}

The age range of the patients was $4 \frac{1}{2}-58$ years with a male-to-female ratio of 1.88:1. The mean total leukocyte count was $115,543 \pm 5462 / \mathrm{mm}^{3}$ and mean blast percentage was $70 \% \pm 12 \%$. Aberrant expression of CD10 (17 cases), CD79a (19 cases), CD33 (13 cases), and CD117 (14 cases) was frequently observed and was further studied for morphological correlation ( 23 cases). Table 1 shows the frequency of these markers. The expression of CD79a was seen in $47.36 \%$. However, it was dim in most of the cases. The data of expression of CD2, CD4, CD5, CD8, CD7, and Tdt were not available in all the cases; hence, the categorization of T-ALL into pro, pre, cortical, and mature Subtypes based on European Group for the Immunological characterization of leukemias (EGIL) could not be done. Further, the aberrant expression was correlated with morphology.

Table 1 shows the percentage aberrant expression of the four markers. Tables 2-5 show the correlation of morphology with aberrant expression of CD10, CD79a, CD33, and CD117, respectively.

- CD117 and CD33-positive cases showed L2 morphology (4/6 [66.6\%] and 4/5 [80\%], respectively) with the presence of convolutions (5/6 [3.3\%] and $5 / 5[100 \%$ ], respectively) [Figure $1 \mathrm{a}$ and Tables 4, 5], while cases with expression of CD79a showed L1 morphology (9/9 [100\%]) with absent to occasionally present convolutions $(9 / 9[100 \%])$ [Figure $1 \mathrm{~b}$ and Table 3].

- CD10 positive cases had L1/L2 morphology with absent to occasionally present convolutions [Table 2].

\section{Discussion}

Acute leukemia is a Clonal expansion of tumor cells in bone marrow, blood, or other tissues. It can be
Table 1: Aberrant expression in percentage

\begin{tabular}{lcc}
\hline Markers & Aberrant expression & Percentage \\
\hline CD10 & $6 / 17$ & 35.3 \\
CD79a & $9 / 19$ & 47.36 \\
CD33 & $5 / 13$ & 38.46 \\
CD117 & $6 / 14$ & 42.28 \\
\hline
\end{tabular}

Table 2: Correlation of morphology with aberrant expression of CD 10 (6/17 [35.3\%])

\begin{tabular}{lcl}
\hline Number of cases & Morphology & Convolutions \\
\hline 3 & L1 & Absent \\
2 & L1 + L2 & Occasional \\
1 & L2 & Present \\
\hline
\end{tabular}

Table 3: Correlation of morphology with aberrant expression of CD79a (9/19 [47.36\%])

\begin{tabular}{lcl}
\hline Number of cases & Morphology & Convolutions \\
\hline 5 & L1 & Absent \\
4 & L1 & Occasional-slight \\
\hline
\end{tabular}

Table 4: Correlation of morphology with aberrant expression of CD117 (6/14 [42.28\%])

\begin{tabular}{lcl}
\hline Number of cases & Morphology & Convolutions \\
\hline 4 & L2 & Present \\
1 & L1 & Present \\
1 & L1 & Absent \\
\hline
\end{tabular}

Table 5: Correlation of morphology with aberrant expression of CD33 (5/13 [38.46\%])

\begin{tabular}{lcl}
\hline Number of cases & Morphology & Convolutions \\
\hline 4 & L2 & Present \\
1 & L1 & Present \\
\hline
\end{tabular}

either acute myeloid leukemia or acute Lymphoblastic leukemia (ALL) based on the lineage of the blast cells under the control of cytokines. Blasts from different lineages express different subsets of surface molecule called as cluster of differentiation (CD) antigens, the expression of which can be determined by flow cytometry. Sometimes, blast cells of one lineage do not exhibit the features of normal cellular differentiation but show the expression of other cell lineage. This phenomenon is called as "aberrant expression" of CD markers. ${ }^{[2]}$

T-ALL is an aggressive malignancy involving peripheral blood, bone marrow, and lymph nodes with frequent involvement of central nervous system. It commonly occurs in children and adolescents, being more common in men than women. Immunophenotypically, T-ALL is positive for $\mathrm{CD} 2, \mathrm{CCD} 3, \mathrm{CD} 7, \mathrm{CD} 34$, and human leukocyte antigen-D related. ${ }^{[3]}$ Aberrant expression of myeloid antigens has been reported in $10 \%-47 \%$ in ALL. ${ }^{[2]}$ In T-ALL, CD10 $(15 \%-40 \%)^{[4]}$ and CD79a $(40 \%)^{[5]}$ are commonly expressed. While CD33 may be expressed $(20 \%-33 \%){ }^{[3]}$ the expression of $\mathrm{CD} 117$ is a rare phenomenon. ${ }^{[3]}$ 


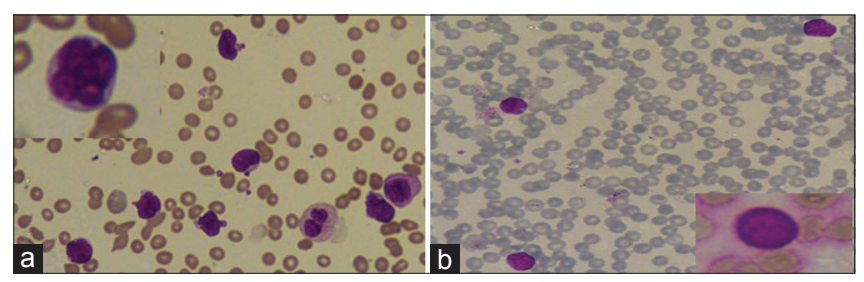

Figure 1: (a) Lymphobalsts with L2 morphology and many convolutions (Giemsa, x400). Inset: a lymphoblast with flower-like convolutions. (b) Lymphoblasts with L1 morphology and absent convolutions (Giemsa, $\times 400$ ). Inset: lymphoblast with absent convolutions

Various studies have reported aberrant antigen expression to be associated with prognostic significance and chromosomal alterations. Bhushan et al. in their study have shown that expression of myeloid antigens CD13 and CD33 has a favorable outcome in T-ALL but not in B-ALL. ${ }^{[6]}$ CD117 is generally considered a marker of leukemic cells committed to the myeloid lineage but rarely can be expressed in T-ALL. Hoehn et al. in their study concluded that the immunophenotypic profile of $\mathrm{TdT} \pm \mathrm{CD} 7 \pm \mathrm{CD} 13 \pm \mathrm{CD} 34 \pm \mathrm{CD} 117+$ (bright) is highly suggestive of FLT3 mutation, with $100 \%$ sensitivity and $94 \%$ specificity in T/myeloid and T-ALL. ${ }^{[7]}$ It has also been suggested that CD117 could be used as a surrogate for FLT3 and CD117-positive T-ALL patients can benefit from FLT3 inhibitors. ${ }^{[8]}$

CD79a is considered a useful marker for identifying B-cell lineage in malignant neoplasms. It is a cell-surface molecule which interacts with membrane immunoglobulins through its cytoplasmic domain and mediates intracellular signal transduction after antigen binding. In view of this interaction, it is considered to be highly specific for B cells. It is also a highly sensitive marker for B cells as it is expressed in the earliest stage of B-cell development, preceding the expression of CD19 as well as in the late stage of B-cell differentiation (i.e., plasma cells). However, aberrant expression of CD79a (40\%) has been reported in T-ALL, suggesting that it might not be as specific as it was believed. ${ }^{[5]}$ Pilozzi et al. performed gene rearrangement studies in ALL patients with co-expression of CCD 3 and CD79a and showed that all the patients studied had $\mathrm{T}$ cell receptor gamma gene rearrangement, and none showed immunoglobulin heavy chain gene rearrangement, strongly suggesting that CD3+ cases of ALL are of T-cell lineage, regardless of CD79a expression. ${ }^{[9]}$ This also points to the fact that CD79a can be expressed independently of B-cell receptor. CD79a-positive T-ALL is confined to pediatric age group ( $\leq 18$ years), are associated with unusual cytogenetic abnormalities (complex karyotypes), as well as respond poorly to treatment as studied by Lai et al. ${ }^{[5]}$

CD10, initially known as common acute lymphoblastic leukemia antigen, was identified as one of the earliest markers expressed by leukemic cells of the lymphoblastic lineage. CD10 expression on leukemic cells remains a useful subclassification (EGIL) tool for B-lineage leukemias, but its aberrant expression is quite commonly seen in T-ALL. Earlier studies have reported CD10 positive T-ALL to have a favorable outcome, ${ }^{[4]}$ but Consolini et al. concluded in his study that CD10 expression does not have any independent prognostic significance T-ALL ${ }^{[10]}$ Furthermore, they found that CD10 T-ALL had FAB L2 morphology. ${ }^{[10]}$

In the present study, we correlated the expression of aberrant immunophenotypic markers with FAB morphological subtypes in T-ALL. CD117 and CD33-positive cases were found to have L2 morphology in $66.6 \%$ and $80 \%$ of positive cases, respectively, with the presence of convolutions in $83.3 \%$ and $100 \%$ of positive cases, respectively [Figure 1a and Tables 4, 5]. Cases with expression of CD79a showed L1 morphology with absent-slight convolutions in $100 \%$ of positive cases [Figure $1 \mathrm{~b}$ and Table 3]. On extensive literature search, we could not find any study correlating the association of aberrant expression of CD117, CD33, and CD79a with FAB morphological subtypes in T-ALL. CD10-positive cases had L1/L2 morphology with absent to occasionally present convolutions [Table 2]. Consolini et al. have correlated CD10 T-ALL with L2 morphology, but we did not find such a correlation in our study. ${ }^{[10]}$

\section{Conclusion}

There seems to be an association of aberrant markers (CD117, CD33, and CD79a) with L1/L2 morphology and absence or presence of convolutions. This is the first study to see the correlation of expression of these three aberrant immunophenotypic markers with morphological subtype in T-ALL. Morphological FAB subtype of T-ALL could point toward the aberrant marker likely to be expressed and hence can guide the hematologist about the prognosis; chromosomal aberrations likely to be associated and the targeted therapy from which the patient is likely to be benefitted. In addition, this can reduce the cost of extensive flow cytometry panels which has its own importance in a developing Indian nation. However, these correlation needs to be tested for statistical significance on a larger sample size and thus pave the way for future research.

\section{Acknowledgments}

The authors acknowledge the Hematology Section of Pathology Department of University College of Medical Sciences and Guru Teg Bahadur Hospital and Max Hospital Saket, New Delhi, for their help in retrieval of case records.

\section{Financial support and sponsorship} Nil. 


\section{Conflicts of interest}

There are no conflicts of interest.

\section{References}

1. Amin MM, Kermani S, Talebi A, Oghli MG. Recognition of acute lymphoblastic leukemia cells in microscopic images using k-means clustering and support vector machine classifier. J Med Signals Sens 2015;5:49-58.

2. Borowitz MJ, Falletta JM. Leukemias and lymphomas of thymic differentiation. Clin Lab Med 1988;8:119-34.

3. Ahmadzadeh A, Saedi S, Jaseb K, Asnafi AA, Alghasi A, Saki N, et al. T-cell acute lymphoblastic leukemia with del (7) (q11.2q22) and aberrant expression of myeloid markers. Int J Hematol Oncol Stem Cell Res 2013;7:40-4.

4. Pui CH, Rivera GK, Hancock ML, Raimondi SC, Sandlund JT, Mahmoud $\mathrm{HH}$, et al. Clinical significance of CD10 expression in childhood acute lymphoblastic leukemia. Leukemia 1993;7:35-40.

5. Lai R, Juco J, Lee SF, Nahirniak S, Etches WS. Flow cytometric detection of CD79a expression in T-cell acute lymphoblastic leukemias. Am J Clin Pathol 2000;113:823-30.

6. Bhushan B, Chauhan PS, Saluja S, Verma S, Mishra AK, Siddiqui S, et al. Aberrant phenotypes in childhood and adult acute leukemia and its association with adverse prognostic factors and clinical outcome. Clin Exp Med 2010;10:33-40.

7. Hoehn D, Medeiros LJ, Chen SS, Tian T, Jorgensen JL, Ahmed Y, et al. CD117 expression is a sensitive but nonspecific predictor of FLT3 mutation in T acute lymphoblastic leukemia and T/myeloid acute leukemia. Am J Clin Pathol 2012;137:213-9.

8. Paietta E, Ferrando AA, Neuberg D, Bennett JM, Racevskis J, Lazarus $\mathrm{H}$, et al. Activating FLT3 mutations in CD117/KIT(+) T-cell acute lymphoblastic leukemias. Blood 2004;104:558-60.

9. Pilozzi E, Müller-Hermelink HK, Falini B, de Wolf-Peeters C, Fidler C, Gatter K, et al. Gene rearrangements in T-cell lymphoblastic lymphoma. J Pathol 1999;188:267-70.

10. Consolini R, Legitimo A, Rondelli R, Guguelmi C, Barisone E, Lippi A, et al. Clinical relevance of CD10 expression in childhood ALL. The Italian association for pediatric hematology and oncology (AIEOP). Haematologica 1998;83:967-73. 